\title{
Un programa de evaluación y comunicación del riesgo cardiovascular reduce el riesgo calculado y los factores de riesgo modificables
}

A cardiovascular risk evaluation and communication program reduces the calculated risk and the modifiable risk factors Benner J y col. Int J Clin Pract. 2008;62(10):1484-98.

\section{Objetivo}

Analizar si un programa de evaluación y comunicación (PEC) del riesgo cardiovascular (RCV) a 10 años (REACH OUT) puede disminuir el riesgo calculado mediante el puntaje de Framingham, a lo largo de seis meses, en comparación con la atención habitual.

\section{Diseño, lugar y pacientes}

Ensayo clínico controlado, aleatorizado por clusters*, multinacional, multicéntrico y abierto; llevado a cabo en cien centros de atención primaria de nueve países europeos (1103 pacientes). Fueron aleatorizados los centros de atención primaria al grupo de atención habitual $(n=461)$ vs. el PEC-RCV $(n=524)$. Fueron incluidos participantes de ambos sexos de 45 a 64 años de edad que presentaran hipertensión arterial y un RCV a diez años calculado por el puntaje de Framingham ${ }^{1}$ mayor a $10 \%$.

\section{Intervención y medición de los resultados principales} Los médicos del grupo intervención calculaban el RCV a diez años del participante y lo asesoraban de acuerdo al riesgo estimado y a los factores de riesgo modificables, teniendo en cuenta los lineamientos del programa. Los médicos del grupo control, desconocían el RCV a 10 años de los participantes por lo que realizaban su práctica habitual. Se analizó por intención de tratar el puntaje el puntaje de Framingham a los seis meses de concluida la intervención.

\section{Resultados Principales}

Luego seis meses, el RCV a 10 años se redujo más en el grupo intervención (OR 0,9; IC95\% 0,84 a 0,96). Ver tabla 1.

Tabla 1: modificación del riesgo cardiovascular estimado a diez a través del puntaje de Framingham.

\begin{tabular}{c|c|c}
$\begin{array}{c}\text { Riesgo cardiovascular } \\
\text { absoluto calculado a través } \\
\text { del puntaje de Framingham }\end{array}$ & $\begin{array}{c}\text { Cuidados habituales } \\
(\mathbf{n = 4 6 1 )}\end{array}$ & $\begin{array}{c}\text { PEC-RCV } \\
(\mathbf{n = 5 2 4})\end{array}$ \\
\hline Riesgo basal (DS) & $16,9 \%(0,26)$ & $17,2 \%(0,25)$ \\
\hline Riesgo a los seis meses (DS) & $13,7 \%(0,27)$ & $12,5 \%(0,25)$ \\
\hline Diferencia ajustada* (IC95\%) & $-4,9 \%(-5,5$ a $-4,3)$ & $-6,3 \%(-6,9$ a $-5,7)$ \\
\hline
\end{tabular}

* Ajustado por país, edad, género y riesgo basal. DS: desvío estándar.

\section{Conclusiones}

El estudio REACH OUT demostró en pacientes con riesgo moderado que el programa de evaluación y comunicación del RCV se asoció a una reducción estadísticamente significativa del RCV a 10 años calculado a través del puntaje de Framingham.

Palabras claves: Riesgo cardiovascular, puntaje de Framingham, factores de riesgo cardiovascular modificables.

Key words: cardiovascular risk, Framingham score, modifiable cardiovascular risk Key words

Fuente de financiamiento: Pfizer Inc.

\section{Comentario}

Las enfermedades cardiovasculares (ECV) son la principal causa de muerte en el mundo, por lo que es importante intervenir sobre los factores de riesgo cardiovasculares modificables como la hipertensión, el tabaquismo y la dislipemia. Sin embrago, las modificaciones en los factores de riesgo son subóptimas en la práctica clínica, entre otras cosas debido a la falta de aplicación de guías de práctica clínica por parte de los profesionales de la salud y a la falta de adherencia al tratamiento por parte de los pacientes. Se necesitan nuevas estrategias a fin de motivar a pacientes y médicos para reducir los factores de riesgo modificables y así lograr una disminución de la ECV. Este estudio, utiliza un novedoso dispositivo, que evalúa e informa el RCV y aconseja al paciente según sus factores de riesgo modificables mostrando beneficios a los seis meses, incluyendo una reducción en los niveles de tensión arterial y tabaquismo. Además, la prescripción de medicamentos aumentó en ambos grupos, lo que probablemente contribuyó a mejorar el pronóstico en ambos grupos.
Vale destacar que las intervenciones dirigidas a mejorar la adherencia del paciente, por ejemplo, el uso de la poli píldora para mejorar la comodidad de la administración, podrían proporcionar beneficios adicionales al control de los factores de riesgo ${ }^{2-4}$.

\section{Conclusiones del comentador}

Varias guías de práctica clínica, hacen hincapié en la importancia de tener en cuenta el RCV global en el manejo de la ECV, ya que los factores de riesgo individuales, son pobres predictores del RCV absoluto. El puntaje de Framingham, da una idea del RCV global de un paciente y puede ser una herramienta útil, accesible, y pasible de ser utilizada por el personal de salud médico y "no médico", que ayudaría en la toma de decisiones y en la promoción cambios saludables en el estilo de vida.

Gabriela Buela [ Servicio de Medicina Familiar y Comunitaria. Hospital Italiano de Buenos Aires. gabriela. buela@ hospitalitaliano.org.ar ]

Buela G. Un programa de evaluación y comunicación del riesgo cardiovascular reduce el riesgo calculado y los factores de riesgo modificables. Evid. actual. práct. ambul; 13(3):91. Ago-Oct. 2010. Benner J y col. A novel programme to evaluate and communicate 10-year risk of CHD reduces predicted risk and improves patients' modifiable risk factor profile. Int J Clin Pract. 2008;62(10):1484-98. PMID: 18691228. Disponible en: http://www.ncbi.nlm.nih.gov/pmc/articles/PMC2658032/?tool=pubmed

\section{Referencias}

1. Wilson P y col. Prediction of coronary heart disease using risk factor categories. Circulation. 1998;97:1837-47.

2. Bramley $\mathrm{T}$ y col. Relationship of blood pressure control to adherence with antihypertensive monotherapy in 13 managed care organizations. $\mathrm{J}$ Manag Care Pharm. 2006;12:239-45

3. Wei $L$ y col Adherence to statin treatment and readmission of patients after myocardial infarction: a six-year follow up study. Heart. 2002;88:229-33.

4. Sokol M y col. Impact of medication adherence on hospitalization risk and healthcare cost. Med Care. 2005;43:521-30. 